XXXI International Workshop on High Energy Physics:

Critical Points in the Modern Particle Physics

International Journal of Modern Physics: Conference Series

Vol. 47 (2018) 1860088 (9 pages)

(C) The Author(s)

DOI: $10.1142 / \mathrm{S} 2010194518600881$

\title{
Searches for Supersymmetry in ATLAS
}

\author{
A. Cervelli (on behalf of ATLAS Collaboration) \\ INFN Sez. di Bologna, \\ V.le C. Berti Pichat 6/2 40127 Bologna, Italy
}

Published 6 June 2018

\begin{abstract}
After the discovery of the Higgs boson in ATLAS first run of data taking, and due to the lack of observation of new physics, searches for new particles such as Supersymmetric states are one of the main area of interest for the general purpose detectors operating at LHC. In this talk we will present a review of the searches for Supersymmetric particles, performed by the ATLAS experiment
\end{abstract}

Keywords: ATLAS; SUSY.

\section{Introduction}

Two of the major highlights in LHC Run 1 by ATLAS ${ }^{1}$ are the discovery of the Higgs boson $^{2}$ and the lack of observation of any physics not predicted by the Standard Model (SM). These results on one hand make our understanding of the SM more complete, on the other do not provide any hint on some open questions. In particular the SM falls short in explaining particle hierarchy (which needs a very fine tuning for Higgs mass stability), does not provide a good candidate for dark matter and fails to provide a unification of coupling constants at high energy scales.

Supersymmetry (SUSY) provides an extension of the SM and it is a framework with a large number of parameters allowing for a large number of possible phenomenologies. SUSY is a time-space symmetry linking each SM particle to a SUSY partner, which has the same properties regarding its interactions, but differs by a half of spin unit (i.e. each SM fermion is bound to have a boson SUSY partner). The introduction of SUSY particles would indeed stabilize the quadratic divergencies arising from loop correction on Higgs boson mass, thus solving the Higgs mass stability problem present in the SM. Since SUSY particles have not been observed at current energies SUSY symmetry should be broken, as the mass of the SM particles and their partners should differ, with SUSY particles mass much higher than the

This is an Open Access article published by World Scientific Publishing Company. It is distributed under the terms of the Creative Commons Attribution 4.0 (CC-BY) License. Further distribution of this work is permitted, provided the original work is properly cited. 


\section{A. Cervelli}

SM counterparts. For SM and SUSY particles it is possible to define the $R$-parity, defined as $R=(-1)^{3(B-l)+2 s}$ (with $\mathrm{B}, \mathrm{l}$, and $\mathrm{s}$, being respectively the baryon and leptonic number, and $\mathrm{s}$ being the spin: $R$ equals 1 for SM particles and -1 for SUSY ones and, if conserved, the lightest SUSY particle (LSP) would provide a natural dark matter candidate.

Among the many phenomenologies predicted by the SUSY framework, in the following we will focus mostly on natural SUSY: ${ }^{3}$ this scenario looks for a fine-tuning as mild as possible, and it is taking into account results from both very large scales (dark matter relic density, cosmological constraints) and sub-atomic scales (Higgs measurements). The natural scenarios typically predict very heavy gluinos (more than $1 \mathrm{TeV}$ masses), a relatively light $3^{\text {rd }}$ generation squarks, and light higgsinos; all other particles are considered to have very high masses and be decoupled with respect to the others, and Higgs partner would be the LSP and the dark matter candidate, in case of $R$-parity conservation.

In ATLAS Run 2, thanks to the increase of center of mass energy in LHC collisions to $13 \mathrm{TeV}$, the predicted cross section for SUSY particle production is expected to increase by a factor between 3 and 10 , while most of the backgrounds components observe a smaller increase in cross section. These two effects combined allow for an expected larger sensitivity to SUSY processes observation. In particular it should be noted that the early data will improve mostly gluino and $1^{\text {st }}$ and $2^{\text {nd }}$ generation particle production, while after $10 \mathrm{fb}^{-1}$ of recorded data, a sizeable improvement in sensitivity is expected for $3^{\text {rd }}$ and electroweakly produced SUSY particles.

There are three main classes of searches which we will cover:

- $R$-parity conserving scenarios, where SUSY particles are produced in pairs through strong or electroweak interaction, and then decay with cascades to the stable LSP. They are characterized by final states with many high $P_{T}$ SM particles and a large missing $E_{T}$ due to LSP escaping detection.

- $R$-parity violating scenarios, where the LSP is not stable anymore, are characterized by multi-jets and multi-lepton signatures from LSP decays, and could show displaced vertexes due to long lived LSP decays.

- Long lived particles: scenarios where SM partners show long lifetimes, mainly due to almost degenerate mass states or small couplings. In this case the signature is given by secondary vertices far from the interaction point, and the analyses are mainly detector performance driven.

\section{Searches Strategy}

The strategy for the SUSY searches is similar for all analysis presented. In general for a given signal model, first a simplified model $^{4}$ is considered: assuming $100 \%$ branching ratios (BR) along the decay cascade for the SUSY particles to the sought after final state. With this rationale the number of free parameters from SUSY model is reduced, and it's easier to both present results in term of SUSY masses, and 
perform a more complex model-dependent reinterpretation for the results at a later stage. The search is optimized either for discovery (in case of inclusive selections or cut-and-count analysis), or for the best exclusion (when more complex selection methods as MVA or shape fits come into play). In each search one or more Signal Regions (SRs) are defined, these regions being those where the signal to background ratio is the highest, and are blinded until the background estimation have been studied and validated.

The backgrounds are grouped in two main classes, whose content may vary from final state to final state: reducible and irreducible backgrounds.

- Irreducible backgrounds are represented by those processes which have signatures which cannot be distinguished from the signal (i.e. produce the same number of leptons, jets, neutral particles etc.). For the most conspicuous backgrounds the estimation obtained from Monte Carlo (MC) simulations is normalized to data in process-enhanced control regions (CR), and the extrapolation is validated in Validation Region (VR) and SR. For non dominant processes the pure MC prediction is used instead.

- Reducible backgrounds arise mainly from mis-reconstruction of particles and detector effects, such as fake leptons (i.e. jets reconstructed as leptons), fake missing $E_{T}$, fake jets, charge flip. The reducible background contribution is taken from purely data driven, or partially data driven, estimates, and are validated in the VR.

When the background modeling and estimates are validated, a simultaneous fit of all background components is performed in both the CRs and the SRs, where the exclusion limit is actually set. An example of how CR, VR, and SR are designed can be found in Fig. 1.

Depending on the final states a wide array of selection variables is used, varying from less complex (such as the multiplicity of leptons, jets, photons, or their energy and momenta), to scale variables, angular variables, mass variables (obtained from combining more reconstructed objects), event shape variables (which use a vast
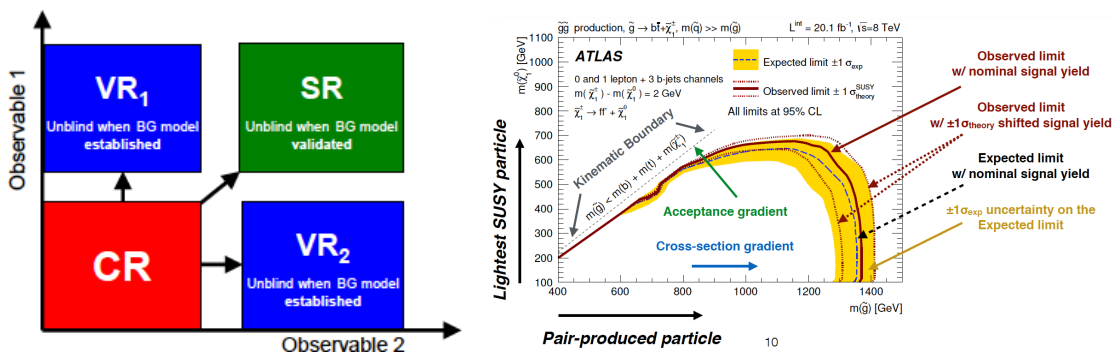

Fig. 1. On the left: a simplified example of CR, VR, SR design for a selection on 2 observables. On the right: an annotated limit plot, showing the limit and its error, the diminishing cross section gradient on the $\mathrm{x}$-axis, and the acceptance gradient running towards the kinematic limit on the diagonal. 


\section{A. Cervelli}

majority of the reconstructed objects), or even variables which are hypotheses based (e.g. $m_{T 2}$ ) and recursive jigsaw reconstruction, ${ }^{5}$ aimed to reconstruct the fourmomenta of states with missing transverse energy.

The results are typically presented in a two dimensional slice of the SUSY particle mass, and the consistency between the signal hypothesis and the background only hypothesis is evaluated as a p-value over discrete points of the plane. An annotated example of a limit is shown in Fig. 1. Model dependent limits are set for each mass point as limits on the production cross section.

\section{ATLAS Run 2 results}

ATLAS performed a wide array of searches using the full dataset available, $36 \mathrm{fb}^{-1}$ from its second run of data taking, performed at a center of mass energy of $13 \mathrm{TeV}$. A complete set of results can be found here. ${ }^{6}$ The analysis can be broken down in four wide categories, for which in the following one analysis will be taken as example and discussed in more detail.

- Inclusive searches deal with a large array of different final states with similar signatures. In the following the search for final states with no leptons, missing transverse energy and less than 6 reconstructed jets ${ }^{7}$ will be presented;

- $3^{\text {rd }}$ generation searches focus on search for SUSY partners of bottom and top quarks, produced through strong interactions. In this paper we will focus on the search of stop decaying in final states with 1 lepton; ${ }^{8}$

- Electroweak production of SUSY particles is searched for mostly in final states with one or more leptons, coming from the decay cascade of gauge boson partners, or lepton partners. As an example the results from the search of electroweakly produced SUSY particles decaying in final states with 2 or three leptons ${ }^{9}$ will be shown;

- RPV scenarios and long lived particles include very different kind of searches, where SUSY do not have a stable LSP, or the quasi-mass degeneracy predicts very long lived particles. A detector driven search is shown to compliment the other analyses in this paper: the search for displaced vertexes associated with missing transverse energy. ${ }^{10}$

\subsection{Inclusive: search for 0 leptons and 2-6 jets}

The inclusive search for final states with no leptons and less than 6 Jets looks mainly for strongly produced SUSY particles decaying directly, or through gauge boson partners, to the SUSY LSP and other SM particles: the processes involved in production and decay can be seen in Fig. 2. The analysis uses two complementary selection strategies. A cut-and-count based analysis with 24 SRs using the effective mass (defined as $m_{\text {eff }}^{2}=P_{T}$ (Jets) ${ }^{2}+E_{T}^{2}$ ) as main discriminant. The 2-3 jets SRs are sensitive to direct squark decays, 4-5 jets to direct gluino decays, 5-6 to gluino or squark decays through charginos, and 2 large-jets region for gluino with high boost 

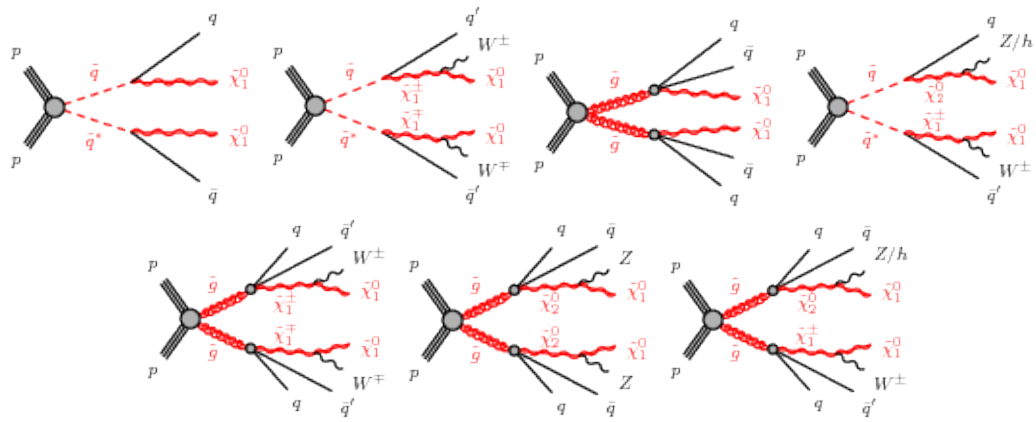

Fig. 2. Decay chains considered in inclusive 0 leptons and 2-6 jets searches.
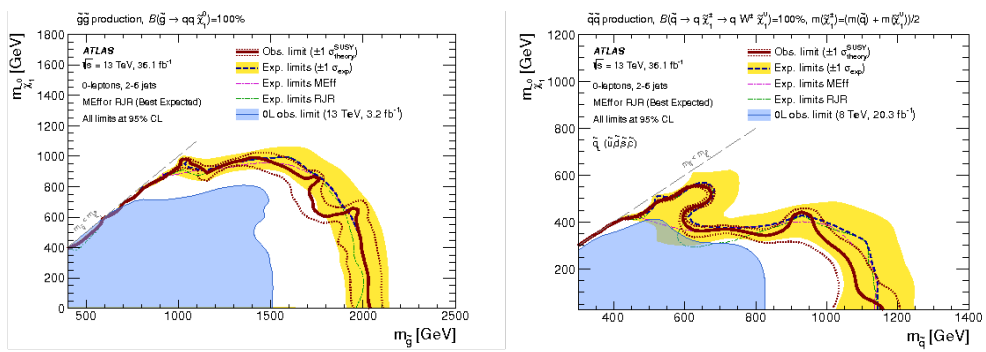

Fig. 3. Exclusion limits for direct gluino decays on the left, and squark decays through chargino on the right. Corresponding respectively to the third and fourth diagrams in Fig. $2 .{ }^{7}$

decaying via charginos: these regions are more sensitive when the mass splitting between the squark or gluino and the LSP is large. The other strategy focuses on the use of recursive jigsaw technique, which infers the four momenta of the invisible particles, which is more sensitive at low mass splittings. The main backgrounds are extrapolated from dedicated $\mathrm{CR}$ and they are: $\gamma+$ jets background (CR defined by adding the $\gamma$ to missing $P_{T}$ ), $\mathrm{W}+$ jets (estimated by considering states with 1 lepton and no b-jets), multi-jets (obtained by reducing the jet resolution from MC), and top (similar to $\mathrm{W}$ CR, but allowing for $1 \mathrm{~b}$-jet). After validating the background estimation, no excess were found in the SRs, so limits were quoted at $95 \%$ Confidence Level (CL). Results for squark pairs decaying through charginos, and direct decays of gluinos are shown in Fig. 3 .

\section{2. $3^{\text {rd }}$ Generation searches: stop 1 lepton}

Stop decays chains depend strongly on both the mass splitting between the $\tilde{t}_{1}$ and the $\chi_{1}^{ \pm}$and the mixing of the gauge boson partners in charginos and neutralinos. These effects are shown in Fig. 4. Different SRs are designed to target different scenarios: in case of pure bino LSP the top, decaying hadronically, is fully reconstructed, and the signal is extracted through a shape fit on missing $E_{T}$. For regions 


\section{A. Cervelli}
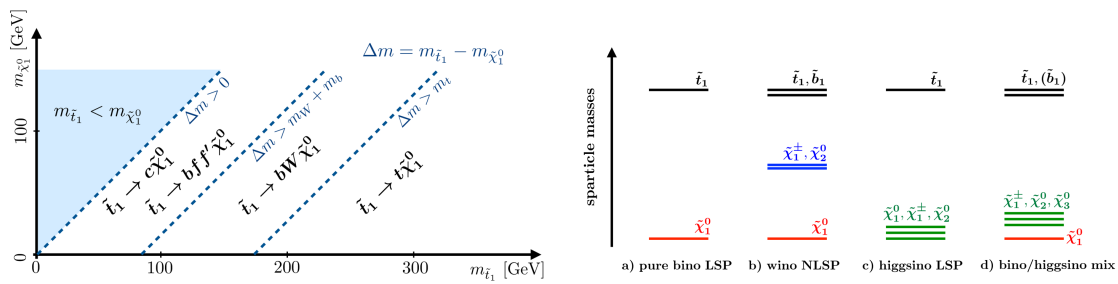

Fig. 4. On the left: the favored decay chain of $\tilde{t}_{1}$, as a function of $\tilde{t}_{1}$ and $\chi_{1}^{0}$ (the LSP). On the right: different phenomenologies in 4 different mixing scenarios for the charginos and neutralinos.

with a small difference in $\tilde{t}_{1}$ and $\chi_{1}^{0}$ masses, the signal selection is performed using multivariate techniques, such as BDT. In case of wino $\chi_{1}^{ \pm}$the favored decay chain is $\tilde{t}_{1} \rightarrow b \chi_{1}^{ \pm}$with $\chi_{1}^{ \pm} \rightarrow W \chi_{1}^{0}$ : in this case the $\mathrm{W}$ is reconstructed from jets. If the LSP is an higgsino instead, a soft spectrum is expected for the jets transverse momenta, and the events are required to have an observed initial state radiation (ISR) jet for boosting the system.

The main background for this analysis are irreducible backgrounds arising from $\mathrm{W}+$ jets and $t \bar{t}$ production, and its rejection is driven mainly by the use of transverse mass, transverse missing energy, and the transverse momentum of the reconstructed W. The 16 signal regions did not show a signal excess, so a set of 95\% CL limits were quoted, and are shown in Fig. 5. With respect to Run 1 a large improvement can be seen for mass splitting close to the top mass, and for splittings smaller than the $\mathrm{W}$ mass.

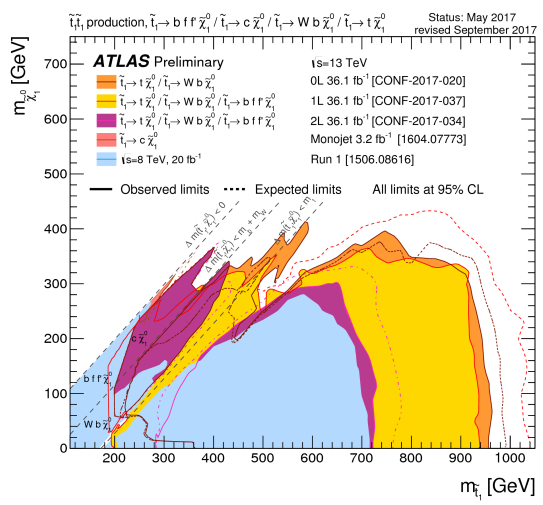

Fig. 5. Summary of the dedicated ATLAS searches for top squark (stop) pair production based on 3.2 to $36 \mathrm{fb}-1$ of pp collision data taken at sqrts $=13 \mathrm{TeV}$. Exclusion limits at 95\% CL are shown in the stop1-neutralino1 mass plane. The dashed and solid lines show the expected and observed limits, respectively, including all uncertainties except the theoretical signal cross section uncertainty (PDF and scale), combining all stop analyses. ${ }^{8}$ 


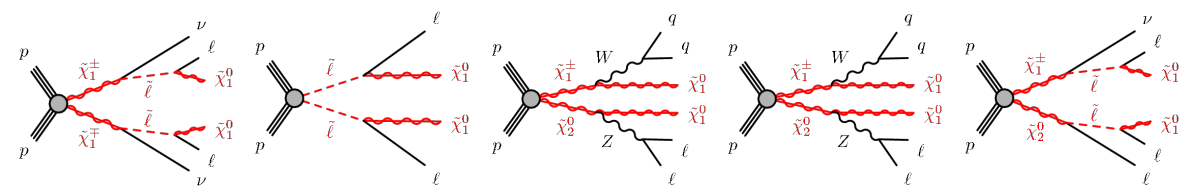

Fig. 6. Decay chains considered in electro weak searches for 2 and 3 lepton final states.
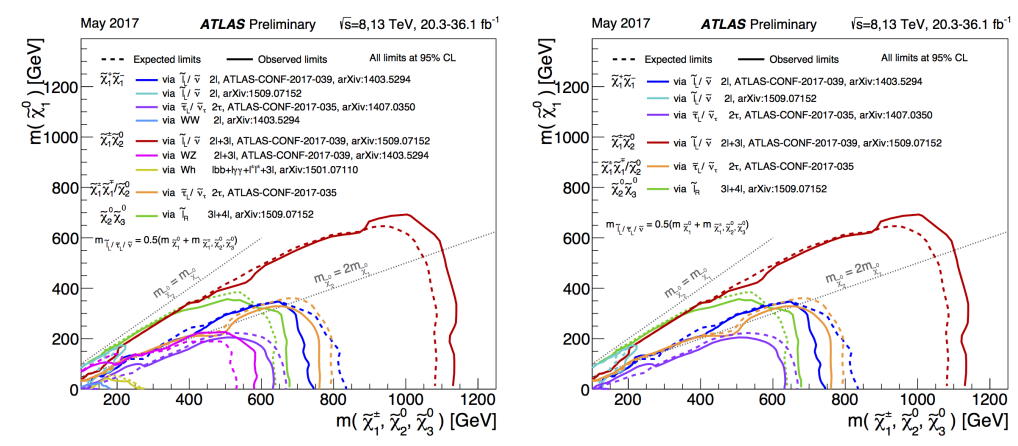

Fig. 7. Decay chains considered in electro weak searches for 2 and 3 lepton final states. ${ }^{9}$

\subsection{Electroweak production: $2 / 3$ lepton final states}

The searches for two and three lepton final states look for pair production of Gauginos decaying via sleptons or bosons, as shown in Fig. 6. Different final state signatures are targeted with different SRs, while their backgrounds, as they are very different, are estimated using different CRs. SRs for 2 leptons, and no jets in final states (the first two scenarios in Fig. 6), target chargino pair production via sleptons; main discriminant is the invariant mass of dilepton pair, and main backgrounds are coming from top pair production and diboson events. The 2 leptons plus jet SRs select mostly chargino-neutralino production, decaying via gauge bosons (third scenarios in Fig. 6), and the background come mostly from $\mathrm{Z}+$ jets events. A 3 lepton selection aims to observe chargino-neutralino production, decaying either via gauge bosons or sleptons (fourth and fifth scenarios in Fig. 6), and backgrounds come from $t \bar{t}$, diboson and single top. No excess was observed, and limits were quoted at $95 \%$ CL. The results of this analysis, combined with all the other electroweak searches, are presented in Fig. 7.

\subsection{Long lived particles: dispalced vertices with missing transverse energy}

Search for displaced vertexes is sensitive to regions of the SUSY framework phasespace inaccessible to other analysis, as scenarios with almost degenerate particles, and small coupling constants. This analysis is mainly detector driven, and targets models where chargino and neutralinos are almost degenerate, and $\chi_{1}^{ \pm}$decaying in 


\section{A. Cervelli}
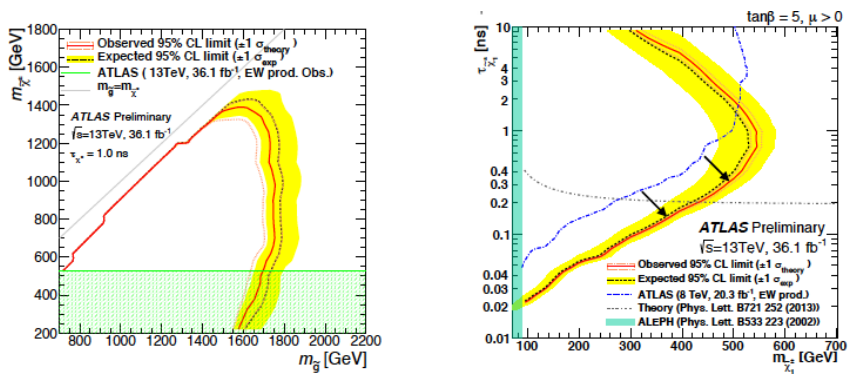

Fig. 8. On the left: limit for strongly produced gliunos. On the right, limits on higgsino like LSP. 10

$\chi_{1}^{0} \pi^{ \pm}$, where the soft pion is lost. Two different signatures are sought after: an ISR jet, with missing $E_{T}$ and a disappearing track, typical for electroweakly produced SUSY particles, or multiple jets, missing $E_{T}$ and a disappearing track, sensitive to strong SUSY production.

The backgrounds from real hadrons or real leptons come from material interaction in the tracker material, and are estimated by smearing the resolution function of the tracker, obtained from MC, to match the disappearing tracks observed in data. Another background come from the fake tracks, where a track is lost due to a wrong reconstruction: this is evaluated from enriched CR, from tracks pointing to the primary vertex, but with no missing transverse energy. No signal excess was found. Limits are reported in Fig. 8: in a pure wino scenarios higgsinos with a lifetime of more than $0.2 \mathrm{~ns}$ and chargino masses up to $460 \mathrm{GeV}$ are excluded. For the strong production channel, gluino masses up to $1.65 \mathrm{TeV}$ are excluded assuming a chargino with $460 \mathrm{GeV}$ mass and a lifetime of $0.2 \mathrm{~ns}$.

\section{Conclusion}

SUSY searches in ATLAS are sensitive to a wide range of new physics scenarios, and are able to look for different physics: strong and weak production, or long lived particles stemming from quasi-degenerate phenomenologies. Different techniques both in terms of high level tools, as multivariate methods or complex kinematic reconstruction, and in detector driven selections were used.

No SUSY particle has been observed yet, but final Run 2 results will provide a better insight on SUSY, thanks not only to the increased statistics recorded, but also thanks to the refinement of the analysis tools. In particular future analyses will be able to target lower cross-section processes, and kinematic regions not accessible with the current statistics.

\section{References}

1. ATLAS Collaboration, 2008 JINST 3 S08003.

2. ATLAS Collaboration, Phys.Lett. B716 (2012) 1-29, arXiv:1207.7214. 
3. J. Alberto Casas, J. Moreno, S. Robles, K. Rolbiecki, B. Zaldivar JHEP 06 (2015) 070

4. J. Alwall, P. Schuster, and N. Toro, Phys.Rev. D79 (2009) 075020, arXiv:0810.3921.

5. P. Jackson, C. Rogan, M. Santoni Phys. Rev. D 95, (2017) 035031

6. https://twiki.cern.ch/twiki/bin/view/AtlasPublic/SupersymmetryPublicResults

7. ATLAS Collaboration, arXiv:1712.02332, submitted to JHEP

8. ATLAS Collaboration, arXiv:1711.11520, submitted to JHEP

9. ATLAS Collaboration, ATLAS-CONF-2017-039

10. ATLAS Collaboration, ATLAS-CONF-2017-026 